\title{
Comunicación
}

\section{Crecimiento y producción de cocones de la Eisenia foetida (lombriz roja) en cuatro sustratos}

\section{Growth and production of cocoons of Eisenia foetida (redworm) on four substrates}

\author{
Ángel Canales-Gutiérrez ${ }^{1,2}$, Nataly Irene Mestas-Gutierrez ${ }^{1}$, \\ Marisol Sheyla Chambi-Alarcon ${ }^{1}$
}

\section{Resumen}

\begin{abstract}
El estudio tuvo como objetivo determinar el crecimiento y número de cocones generados por Eisenia foetida (lombriz roja) en cuatro sustratos. Los tratamientos (sustratos) fueron: choclo (T1), zapallo (T2), camote (T3) y papa (T4) en $1.5 \mathrm{~kg}$ de tierra agrícola, 150 $\mathrm{g}$ de estiércol de alpaca y 25 unidades de rocas medianas. Se utilizaron 15 lombrices rojas adultas por tratamiento, colocadas en cajas de madera y se hizo el seguimiento durante 40 días. Se midió el tamaño y número de lombrices, así como el conteo de cocones. La longitud de las lombrices fue similar para los tratamientos con choclo $(5.5 \mathrm{~cm})$, papa $(5.5$ $\mathrm{cm})$ y zapallo $(5.2 \mathrm{~cm})$, pero menor en el tratamiento con camote $(4.0 \mathrm{~cm} ; \mathrm{p}=0.0045)$. Por otro lado, no hubo diferencia significativa en el número de cocones entre tratamientos.
\end{abstract}

Palabras clave: cocones, crecimiento, Eisenia foetida, residuos orgánicos

\footnotetext{
${ }^{1}$ Programa de Ecología, Escuela Profesional de Biología, Universidad Nacional del Altiplano de Puno, Perú

${ }^{2}$ E-mail: acanales@unap.edu.pe
}

Recibido: 9 de marzo de 2021

Aceptado para publicación: 14 de septiembre de 2021

Publicado: 27 de octubre de 2021

CLos autores. Este artículo es publicado por la Rev Inv Vet Perú de la Facultad de Medicina Veterinaria, Universidad Nacional Mayor de San Marcos. Este es un artículo de acceso abierto, distribuido bajo los términos de la licencia Creative Commons Atribución 4.0 Internacional (CC BY 4.0) [https:// creativecommons.org/licenses/by/4.0/deed.es] que permite el uso, distribución y reproducción en cualquier medio, siempre que la obra original sea debidamente citada de su fuente original 
The aim of this study was to determine the growth and number of cocoons produced by Eisenia foetida (red worm) in four substrates. The treatments (substrates) were corn (T1), pumpkin (T2), sweet potato (T3) and potato (T4) in $1.5 \mathrm{~kg}$ of agricultural soil, $150 \mathrm{~g}$ of alpaca manure and 25 units of medium-size rocks. In total, 15 adult red worms were used per treatment, placed in wooden boxes and monitored for 40 days. The size and number of worms were measured, as well as the cocoons count. The length of the worms was similar for the treatments with corn $(5.5 \mathrm{~cm})$, potato $(5.5 \mathrm{~cm})$ and pumpkin $(5.2 \mathrm{~cm})$, but shorter in the treatment with sweet potato $(4.0 \mathrm{~cm} ; \mathrm{p}=0.0045)$. On the other hand, there was no significant difference in the number of cocoons between treatments.

Key words: coconuts, growth, Eisenia foetida, organic wastes

\section{INTRODUCCIÓN}

Muchos suelos se encuentran contaminados o degradados por su sobreexplotación, sobrepastoreo, y uso de químicos como hidrocarburos y fertilizantes, entre otros. Así mismo, la deficiente disposición y tratamiento de los residuos orgánicos viene generando focos infecciosos de vectores de enfermedades, producción de lixiviados y olores inadecuados y, por tanto, degradación de ecosistemas. Por otro lado, se conocen aproximadamente 8000 variedades de lombrices de tierra, de las cuales 3500 han sido estudiadas y clasificadas, pocas de ellas adaptadas para su producción en criaderos (Campoverde et al., 2020). La mayoría de las lombrices de tierra están adaptadas a un hábitat específico; sin embargo, la especie Eisenia foetida (lombriz roja) puede ser utilizada fuera de su hábitat natural (MorenoReséndez y Cano-Ríos 2002).

Como producto de la reproducción sexual se generan los cocones que son capullos o cápsulas de color amarillo verdoso de 2-3 mm de diámetro. Estos contienen un líquido donde se desarrollan los embriones (Fuentes, 1987; Rivera-Arredondo et al., 2018), los cuales están relacionados directa- mente con la disponibilidad de alimento (López-Méndez et al., 2013).

La lombriz roja puede tener múltiples usos, entre ellos la producción de vermicomposta, la que realiza en su tubo digestivo a través de un proceso de biotransformación (Acosta-Durán et al., 2013; Rodríguez et al., 2018), produciendo un fertilizante orgánico con nutrientes que son indispensables para las plantas, siendo una alternativa para el incremento de la capacidad productiva del suelo y resiliencia de los agro ecosistemas (VidañaMartínez et al., 2017; Flores-Pacheco et al., 2018), ya que destaca la capacidad de absorción de agua y favorece la germinación de las plántulas (Ortega-Martínez et al., 2010).

La lombriz roja también puede ser utilizada como una estrategia de biorremediación, debido a que tiene la capacidad de modificar las propiedades químicas y físicas del suelo (Rodríguez et al., 2018); asimismo, favorece el aumento de la comunidad bacteriana en el suelo tratado, produciendo una inmovilización de metales pesados y una adaptación de los microorganismos en un suelo contaminado (Zapata et al., 2017). Por otro lado, puede ser utilizada para el tratamiento de aguas residuales domésticas por un proceso de tratamiento en estructuras denominadas lombrifiltro (Cáceres et al., 2018). 
Dadas sus cualidades benéficas y tomando en cuenta su facilidad de reproducción, ya que puede producir grandes cantidades de lombrices por año (Paco et al., 2011), se requiere adquirir mayores conocimientos sobre la influencia del tipo de alimentación en el crecimiento y producción de cocones de la lombriz roja. Por tanto, el objetivo de esta investigación fue analizar el crecimiento y el número de cocones generados por la lombriz roja en cuatro sustratos.

\section{Materiales y Métodos}

La investigación se realizó en el invernadero de la Oficina de Calidad de Gestión Ambiental Sostenible (OCGAS) de la Universidad Nacional del Altiplano de Puno, a una altitud de $3810 \mathrm{msnm}$. La medición de las lombrices y el conteo del número de cocones se hizo al inicio de experimento (día 0 ), y a los 20 y 40 días para determinar el aumento de longitud de las lombrices, presencia de clitelos y producción de cocones.

Se utilizaron 60 lombrices rojas jóvenes ( $\sin$ clitelo), de $4.5 \mathrm{~cm}$ de promedio de longitud (el tamaño adulto en capacidad reproductiva es de 5-6 cm), obtenidas del invernadero de la OCGAS.

Se utilizaron 15 lombrices por tratamiento en contenedores de madera $(0.50 \times 0.30 \mathrm{x}$ $0.15 \mathrm{~m})$. En cada tratamiento se colocó 1.5 $\mathrm{kg}$ de tierra agrícola, $150 \mathrm{~g}$ de estiércol de alpaca y para la aireación del sustrato se utilizaron 25 piedras de aproximadamente 2.5 $\mathrm{cm}$ de diámetro. En cada sustrato se agregó como material orgánico y según el tratamiento $200 \mathrm{~g}$ de choclo (Zea maíz) (T1), $200 \mathrm{~g}$ de zapallo tipo pumpkin (Cucurbita sp.) (T2), $200 \mathrm{~g}$ de camote (Ipomoea batatas) (T3) o $200 \mathrm{~g}$ de papa (Solanum tuberosum) (T4). El material orgánico fue colocado en forma de trozos de $4 \mathrm{~cm}$ aproximadamente y se encontraban en estado de madurez hortícola. Los contendedores fueron regados diariamen- te con $200 \mathrm{ml}$ de agua para mantener la humedad. En los sustratos no se realizaron procesos de pre-compostaje, debido a que la altura no fue mayor a los $30 \mathrm{~cm}$.

Para efecto de los registros, se vació todo el sustrato de cada contenedor sobre una tabla, en donde se separaron las lombrices rojas y los cocones. Para las mediciones de longitud $(\mathrm{cm})$ de las lombrices se utilizó una regla de $30 \mathrm{~cm}$. El conteo del número de lombrices y cocones se realizó por observación directa.

Se utilizó un diseño experimental con cuatro tratamientos (sustratos). Los datos se analizaron con el programa estadístico Infostat 2021, mediante un análisis de varianza. La comparación de medias se hizo con la prueba de Tukey $(\mathrm{p}<0.05)$, para la diferencia de la producción del número de cocones se realizó el análisis de Kruskal Wallis y para la proyección entre la producción del número de cocones con relación al tiempo (60 días) se realizó el análisis de regresión.

\section{Resultados y Discusión}

La media de la longitud de las lombrices a los 40 días del estudio fue similar para los tratamientos con choclo $(5.5 \mathrm{~cm})$, papa $(5.5 \mathrm{~cm})$ y zapallo $(5.2 \mathrm{~cm})$, siendo significativamente menor en el tratamiento con camote $(4.0 \mathrm{~cm} ; \mathrm{p}=0.0045)$ (Figura 1$)$.

Se conoce que el tipo de sustrato influencia el aumento del tamaño de las lombrices rojas. Por ejemplo, Gallardo et al. (2018) utilizando broza de café con cáscara de huevo logró un aumento en la tasa de crecimiento, en tanto que Díaz et al. (2008) utilizando como sustrato el estiércol de bovino y cepa de caña y de plátano favoreció la dinámica de crecimiento y producción de la lombriz roja. 


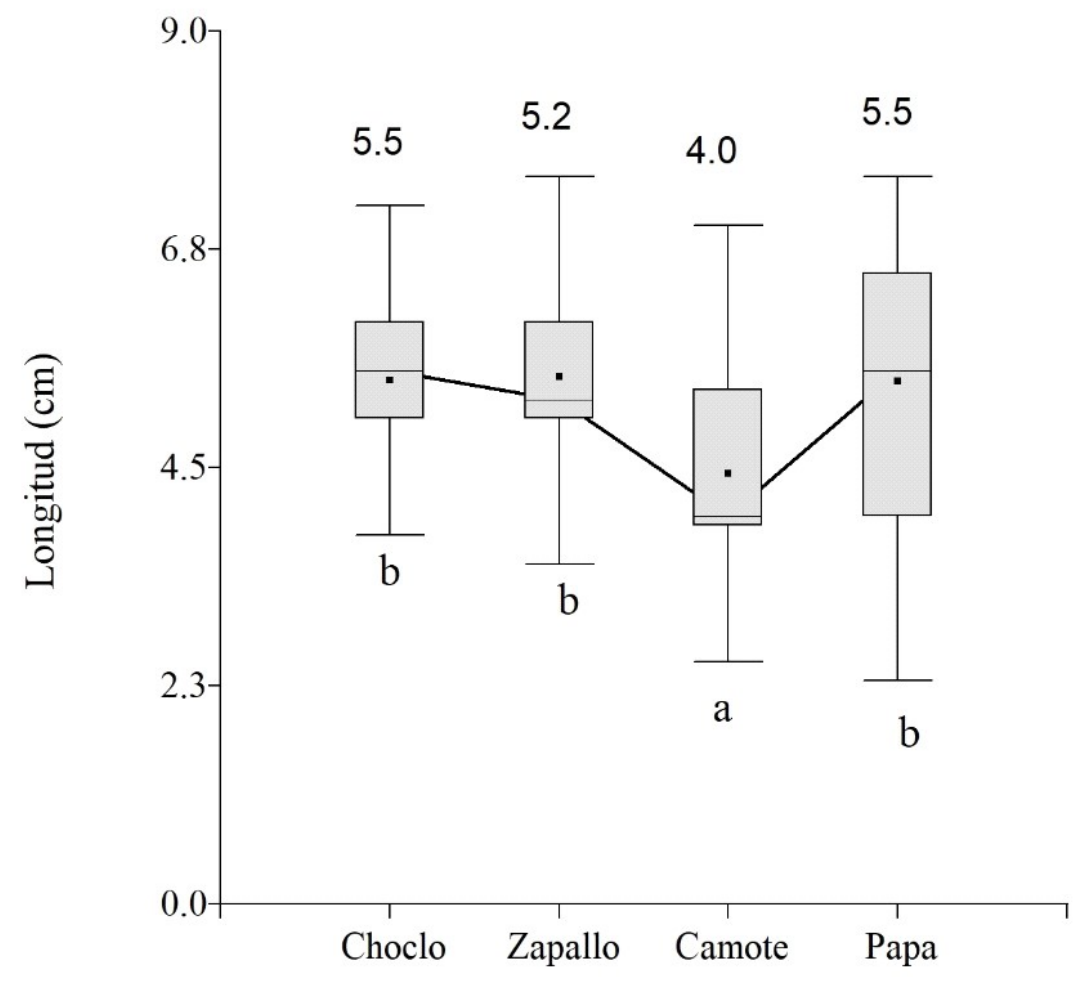

Tratamientos

Figura 1. Medias (cm) de longitud de la lombriz roja (Eisenia foetida) mantenida en cuatro sustratos. Medias con letra diferente son significativamente diferentes $(\mathrm{p}<0.05)$

No hubo diferencia en el crecimiento de la lombriz por efecto de los tratamientos con papa y choclo. Se conoce que el sustrato de cáscara de papa es el más adecuado, obteniéndose mayor cantidad y peso de lombrices (Campoverde et al., 2020). En el presente estudio, el sustrato con choclo no tuvo una degradación rápida, ya que no se dieron las condiciones necesarias para iniciar la hidrólisis que el biopolímero del maíz requiere para disminuir su masa molecular y hacerlo susceptible al ataque microbiológico y/o a la digestión por la lombriz (Ruiz et al., 2013).

Con relación al número de cocones, no se encontró diferencia significativa por efecto de los sustratos $(\mathrm{p}=0.9204)$ (Figura 2). No obstante, otros estudios demuestran diferencias en el número de cocones y tasa de sobrevivencia según el sustrato utilizado (Gutierrez et al., 2007; Gastón et al., 2008; Limachi, 2018). Por ejemplo, los sustratos de broza de café, estiércol de animales y cáscaras de fruta, registraron los mayores valores en reproducción de cocones y sobrevivencia con relación a otros tratamientos (Durán y Enríquez, 2009; Canales et al., 2020).

Al realizar el análisis de regresión para una proyección de 60 días, se pudo determinar que existe una relación de $76 \%$ entre tiempo (días) y número de cocones de la lombriz roja; es decir, el $76 \%$ del número de cocones depende del transcurso del tiempo, mientras que un $24 \%$ podría depender de factores biológicos o reproductivos. Por ejemplo, la lombriz roja puede producir 92 cocones en 60 días $\left(\mathrm{R}^{2}=0.76, \mathrm{a}=-6.67, \mathrm{~b}=1.64\right)$, donde esta- 


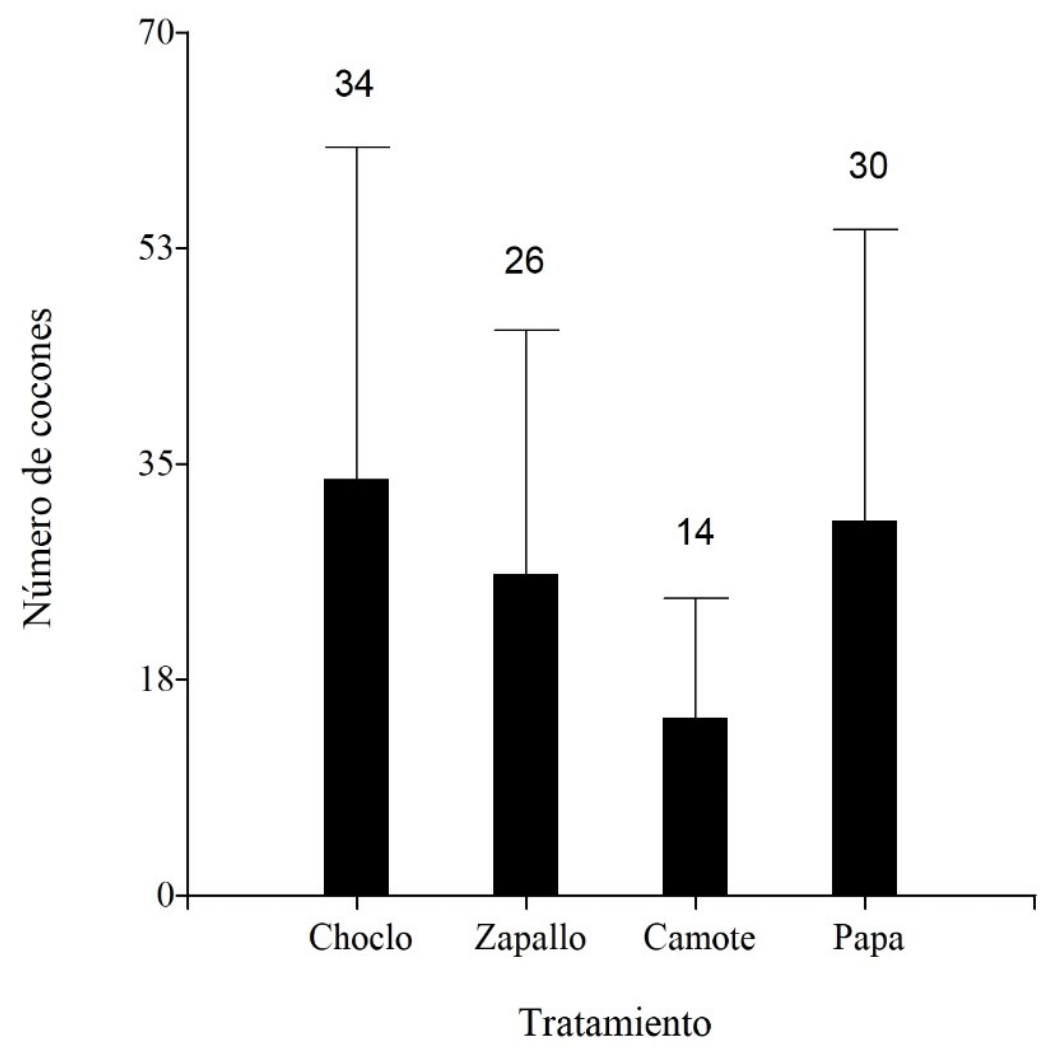

Figura 2. Producción de cocones de lombriz roja (Eisenia foetida) en cuatro sustratos

ría alcanzando su nivel máximo de generación de huevos, toda vez que transcurrido los 70 días, es tiempo suficiente para que las lombrices cumplan su ciclo reproductivo (Campoverde et al., 2020).

\section{Conclusión}

El tratamiento con sustrato de choclo y papa registraron una mayor longitud de crecimiento y producción de cocones, seguida por el tratamiento con sustrato de zapallo.

\section{Literatura Citada}

1. Acosta-Durán CM, Solís-Pérez O, Villegas-Torres OG, Cardoso-Vigueros L. 2013. Precomposteo de residuos or- gánicos y su efecto en la dinámica poblacional de Einsenia Foetida. Agron Costarric 37: 127-139. doi: 10.15517/ rac.v37i1.10718

2. Cáceres DK, Calisaya GM, Bedoya $\boldsymbol{E V}$. 2018. Eficiencia de la lombriz roja californiana (Eisenia foetida) en el tratamiento de aguas residuales domésticas. Rev Cien Tecnol Desarrollo 4: 13-23.

3. Campoverde DK, Velasco LA, Acurio WD. 2020. Aplicación de sustratos orgánicos en la cría de la lombriz roja californiana (Eisenia foetida) para la producción de alimento animal. Conciencia Digital 3: 22-35.

4. Canales A, Solís BJ, Panca R, Quispe B. 2020. Crianza de Eisenia foetida (lombriz roja) en diferentes sustratos de desarrollo biológico. Ecol Apl 19: 87-92. doi: 10.21704/rea.v19i2.1559 
5. Díaz D, Cova LJ, Castro A, García DE, Perea F. 2008. Dinámica del crecimiento y producción de la lombriz roja californiana (Eisenia foetida Sav.) en cuatro sustratos a base de estiércol bovino. Agric Andina 15:39-55

6. Durán L, Henríquez C. 2009. Crecimiento y reproducción de la lombriz roja (Eisenia foetida) en cinco sustratos orgánicos. Agron Costarric 33: 275-281.

7. Flores-Pacheco JA, Romero O, Vivas E, Lacayo J, Cassells R. 2018. Evaluación de la viabilidad de distintas dietas para la producción de lombrihumus con las especies Eisenia foetida y Eudrillus sp. Nexo Rev Cient 31: 2846. doi: 10.5377/nexo.v31i01.6452

8. Fuentes JL. 1987. La crianza de la lombriz roja. Hojas divulgadoras. Madrid, España: Ministerio de Agricultura, Pesca y Alimentacion. $27 \mathrm{p}$.

9. Gallardo L, Contreras Y, Jaramillo J, Alban RE. 2018. La lombriz roja californiana (Eisenia foetida) un aliado en la agricultura urbana sustentable. Cuadernos de Agroecología 13: 1-5.

10. Gastón J, Martínez MM, Cordón E. 2008. Evaluación de la eficacia de cuatro dietas alimenticias sobre el crecimiento, desarrollo y producción de abono de la lombriz californiana (Eisenia foetida). Ciencia e Interculturalidad 2: 67-81. doi: 10.5377/rci.v2i2.573

11. Gutierrez E, Juárez A, Mondragón AJ, Rojas AL. 2007. Dinámica poblacional de la lombriz Eisenia foetida en estiércol composteado y fresco de bovino y ovino. REDVET 8(7). [Internet]. Disponible en: https:// www.redalyc.org/articulo.oa? id=63612662014

12. Limachi E. 2018. Efecto de tres dosis de sustratos en la alimentación de la lombriz roja californiana (Eisenia foetida) con estiércol bovino y aserrín descompuesto en Sapecho, Alto Beni. Apthapi 4: 1328-1138.

13. López-Mendez C, Ruelas-Ayala RF, Sañudo-Torres RR, Armenta C, FélixHerrán J. 2013. Influencia de diferen- tes sustratos orgánicos en la lombriz roja californiana (Eisenia foetida). Tecnociencia Chihuahua 7(2): 81-87.

14. Moreno-Reséndez A, Cano-Ríos P. 2002. Tasa reproductiva de la lombriz roja (Eisenia foetida) en diferentes sustratos orgánicos. Rev Chapingo Serie Zonas Áridas 3: 41-46.

15. Ortega-Martínez LD, Sánchez-Olarte J, Díaz-Ruiz R, Ocampo-Mendoza J. 2010. Efecto de diferentes sustratos en el crecimiento de plántulas de tomate (Lycopersicum esculentum Mill). Ra Ximhai 6: 365-372.

16. Paco G, Loza-Murguía M, Mamani F, Sainz H. 2011. Efecto de la lombriz roja californiana (Eisenia foetida) durante el compostéo y vermicomposteo en predios de la estación experimental de la unidad académica campesina carmen pampa. J Selva Andina Res Soc 1: 24-39.

17. Rivera-Arredondo M, Calderon-Ruiz A, Felix-Morales V, Gaytan-Ruelas M. 2018. Producción in vitro de cocones de la especie Eisenia foetida en diferentes sustratos. Rev Sistemas Exp 5(14): 7-11.

18. Rodríguez F, León D, Quiñones FL, Madrid M, Montoya T, Ordaz L, Tovalin E. 2018. Supervivencia de la lombriz roja (Eisenia foetida) en suelo contaminado con concentraciones de hidrocarburo. Rev Latinoam Ambiente Ciencias 9: 1425-1433.

19. Ruiz M, Pastor K, Acevedo A. 2013. Biodegradabilidad de artículos desechables en un sistema de composta con lombriz. Inf Tecnol 24: 47-56.

20. Vidaña-Martínez SA, HernándezLopéz M, Muro-Jiménez AY. 2017. Evaluación de tres diferentes sustratos para el desarrollo de lombriz roja californiana (Eisenia foetida ). Rev Cienc Ing Desarrollo Tec Lerdo 3: 147-151.

21. Zapata IC, Martínez L, Posada E, González ME, Saldarriaga JF. 2017. Efectos de la lombriz roja californiana (Eisenia foetida) sobre el crecimiento de microorganismos en suelos contaminados con mercurio de Segovia, Antioquia. Cien Ing. Neogranadina 27: 77-90. 